\title{
A NEURAL NETWORK MODEL OF AN ISING SPIN GLASS
}

\author{
K.F. WILSON AND D.J. GoOSSENS \\ Department of Physics, Monash University, Clayton 3168, Australia
}

The behaviour of an Ising spin glass $(S=1 / 2)$ with infinite range interactions is modelled using a numerical simulation based on a neural network. Thermodynamic variables are defined on the network, and are found to obey the Thouless-Anderson-Palmer theory when the applied magnetic field is zero. When a magnetic field is applied along the spin direction, complex field-dependent behaviour appears, including a state in which the Edwards-Anderson order parameter is independent of temperature below the critical temperature.

PACS numbers: 75.10.-b, 75.10.Nr, 84.35.+i

\section{Introduction}

The statistical mechanics of spin glasses have been carried over into the study of neural networks [1]. Here, we explore neural networks as a means of modelling the spin glass phase. Thermodynamic variables such as magnetic susceptibility, magnetisation, and internal energy are defined for the neural network, and their evolution explored as functions of applied field and temperature. We analyse the neural network behaviour in light of the work of Thouless, Anderson, and Palmer [2], which has become of interest in neural networks [3] and which is of continuing interest in physics.

A neural network model is computationally simple with few assumptions. The network used was the Hopfield model [1] with infinite range symmetric interactions. The network consisted of $N$ units with $J_{i j}=J_{j i}$ for $i, j=1,2, \ldots, N$. Each unit in the network had two possible states, \pm 1 . The initial network state was created by storing randomly generated patterns of $N$ bits $\xi_{i}^{\mu}(i=1,2, \ldots, N$; $\mu=1,2, \ldots, p)$, where $p$ was the number of patterns to be stored. The $J_{i j}$ were then generated by [4]

$$
J_{i j}=\sum_{\mu=1}^{p} \xi_{i}^{\mu} \xi_{j}^{\mu}
$$


The energy was calculated from a conventional spin Hamiltonian of the form

$$
H=-\frac{1}{2} \sum_{i j} J_{i j} S_{i} S_{j}-\sum_{j} h^{\mathrm{ext}} S_{j}
$$

where $h^{\text {ext }}$ is the external field and $S_{i}$ is the state \pm 1 of the $i$-th unit.

The simulations were written in C++ on Silicon Graphics R1000 computers, with $N=50,200$ or 800 . All units were updated simultaneously at each time step. Networks were allowed to evolve according to Glauber dynamics [5] for 200 time steps, with magnetisation, $M$, susceptibility, $\chi$, energy, $H$, and Edwards-Anderson order parameter, $q[6]$ being calculated at each step.

The thermodynamic variables were defined on the network as follows:

$$
\begin{aligned}
& M=\frac{2}{N} \sum_{i=1}^{N} S_{i}, \\
& q=\left\langle S_{i}(t) S_{i}(t+1)\right\rangle, \\
& \chi=\lim _{h_{\text {ext } \rightarrow 0}} \frac{M}{h^{\text {ext }}}
\end{aligned}
$$

with energy being given by Eq. (2). Equation (4) was used rather than $\left\langle S_{i}(0) S_{i}(t)\right\rangle$ because it does not depend on the network initial state, and the synchronous updating caused the network to relax very rapidly.

\section{Results and discussion}

At $h^{\text {ext }}=0$, all quantities were found to obey the predictions of the theory developed by Thouless, Anderson, and Palmer (TAP) [2] for $T \leq T_{\mathrm{c}} / 2$, with $\chi$ depending linearly on $T$ and $q$ falling off with $T^{2}$. The TAP parameter, $\alpha$, was found to be $1.4 \pm 0.3$, agreeing with the expected value of 1.665 [2]. The entropy was calculated by integrating $T^{-1} \mathrm{~d} H$, and agreed with expectation, with a limiting value at high temperatures of 0.64 - very close to the expected value of 0.67 [7]. Hence, the simulation is useful quantitatively as well as qualitatively.

Close to $T_{c}, q$ does not go to zero. Instead, $q$ changes continuously from obeying the TAP prediction at small $T$ to being described by $q=M^{2}$ at $T \geq T_{\mathrm{c}}$. This was found to be independent of network size for all sizes investigated, suggesting that the transition is intrinsically a high order transition rather than a first order transition broadened by finite size effects.

The behaviour of $q$ with $T$ is shown in Fig. 1 for various $h^{\text {ext }}$. As $h^{\text {ext }}$ increases, $q$ falls more quickly with $T$, until at the critical field, $h_{c}$, it is constant at 0.5 up to $0.8 T_{\mathrm{c}}$. This state occurs because the system is on the border between falling into a spin glass state and becoming aligned with the applied field. Increasing temperature makes the energy landscape of the network insignificant, so that the only effective ordering is due to the applied field. This effect also falls off with temperature, so that $q$ approaches zero as $T$ increases. As $h^{\text {ext }}$ exceeds $h_{\mathrm{c}}$, it becomes strong enough to align the spins in the network regardless of the intrinsic interactions, and so $q$ becomes large and stays large, although again falling as $T$ increases. For fields greater than $h_{\mathrm{c}}$, no spin glass phase is present, and the system 


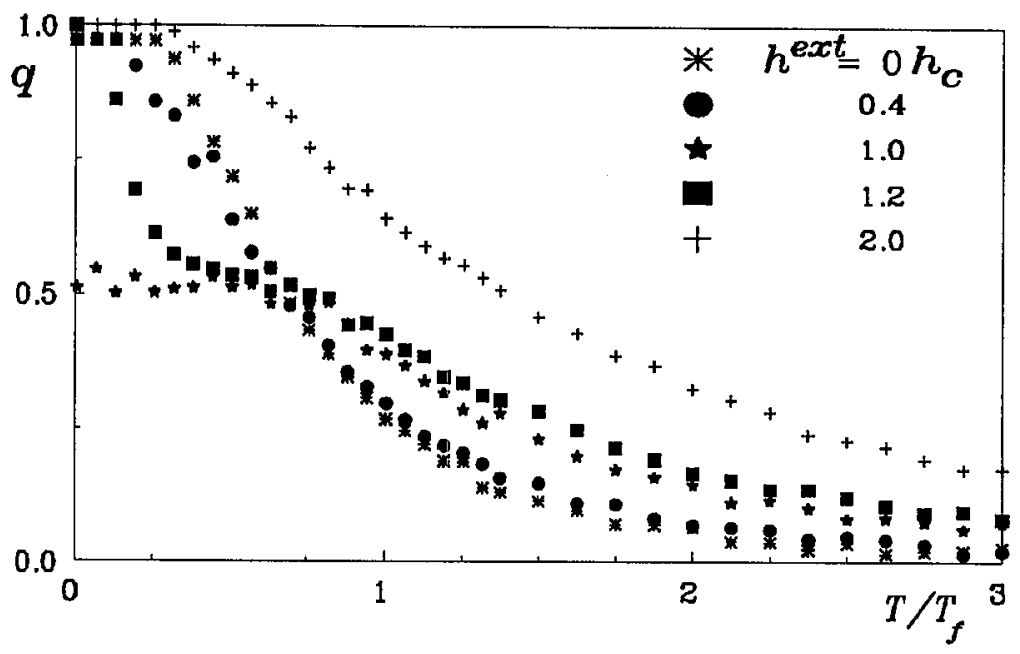

Fig. 1. $q$ as a function of $T$ at various applied fields.

is paramagnetic, with the spins aligned at low temperatures and becoming more disordered as $T$ is increased.

This sort of behaviour is due to competition between $J_{i j}$ and $h^{\text {ext }}$. At low $T$ and $h^{\text {ext }}$, the system sits in a local energy minimum governed by $J_{i j}$, with $T$ too small to bump the system out of that state. As $T$ increases, the system is thermally excited out of the local minimum and the spins are aligned by $h^{\text {ext }}$; this effect increases with $T$. At $T \geq T_{\mathfrak{c}}$, the system behaves paramagnetically.

Behaviour similar to that seen here was observed in [7], but we need no complicated energy minimisation techniques, and make no assumptions beyond the structure of the network. A further advantage of a neural network simulation is that neural network concepts can be used to explore the behaviour of the spin glass model. The idea of content addressable memory involves giving the network part of a stored pattern and allowing it to evolve in order to retrieve the entire pattern. Pattern recall can be measured by a bitwise comparison of the final network state with the stored patterns. Recall falls to zero at the spin glass $T_{\mathrm{c}}$ and $h_{\mathrm{c}}$, because the recall phase of the neural network coincides with the spin glass phase of the spin
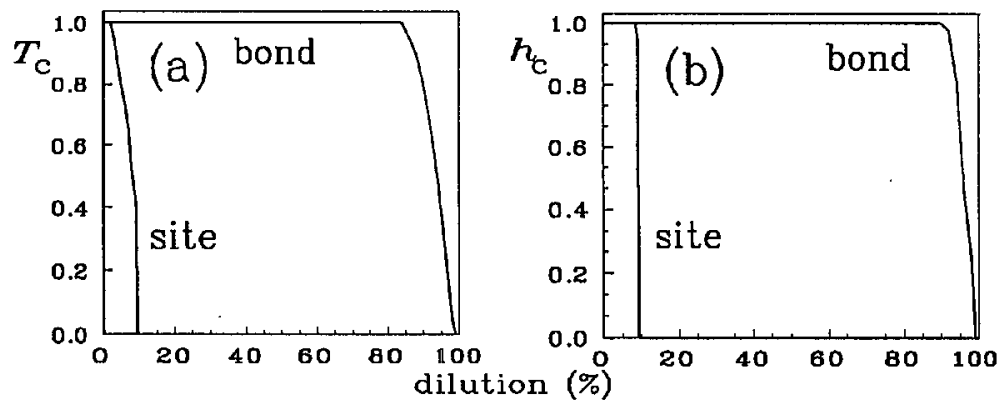

Fig. 2. (a) $T_{\mathrm{c}}$ and (b) $h_{\mathrm{c}}$ as they depend on dilution. 
system. Hence, the concept of recall can be used in exploring the phase behaviour of the system. As recall cannot be tested if $J_{i j}$ are set randomly using a statistical distribution, such an exploration is not possible using a conventional numerical spin glass model.

For example, the effect of dilution was explored. As expected for a system with infinite range interactions, the spin glass phase was very resistant to bond dilution, persisting until over $98 \%$ of bonds were removed (Fig. 2). $T_{c}$ was independent of bond dilution until over $80 \%$ of bonds were removed, and $h_{\mathrm{c}}$ was even more robust, remaining unchanged until over $90 \%$ of bonds were cut. Site dilution removes the units which store the patterns, rather than the links between them, so it is reasonable to expect it to have a stronger effect on recall.

The phase transition with applied field, in which $T$ is held constant and the applied field varied, is first order at $T=0$, but becomes second or higher order at non-zero temperatures. This is logical, as setting $T=0$ removes statistical uncertainty from the simulation.

\section{Conclusions}

Thermodynamic variables can be defined sensibly on a neural network, and can be used to explore the behaviour of that network. The Hopfield net with infinite range, symmetric interactions follows the theory of Thouless, Anderson, and Palmer at low temperatures, and therefore acts as a model of an Ising spin glass. The spin glass to paramagnetic phase transition is of high order, and depends at most weakly on system size.

Neural network concepts such as memory recall were used to explore the spin glass model. It was found that the critical field is more robust against dilution than the critical temperature. The model showed a great deal of complex behaviour, including a state in which the Edwards-Anderson order parameter is independent of temperature, yet different from zero or unity.

This work forms the basis of an exploration into more complex spin glass and neural network models.

\section{Acknowledgments}

The authors are grateful recipients of Australian Postgraduate Awards. D.J.G. thanks AINSE for financial support.

\section{References}

[1] J.J. Hopfield, Proc. Natl. Acad. Sci. USA 79, 2554 (1982).

[2] D.J. Thouless, P.W. Anderson, R.G. Palmer, Philos. Mag. 35, 593 (1977).

[3] A. Theumann, Phys. Rev. E 53, 6361 (1996).

[4] D.O. Hebb, Organisation of Behaviour, John Wiley and Sons, New York 1949.

[5] R.J. Glauber, J. Math. Phys. 4, 294 (1963).

[6] S.F. Edwards, P.W. Anderson, J. Phys. F 5, 965 (1975).

[7] S. Kirkpatrick, D. Sherrington, Phys. Rev. B 17, 4384 (1978). 